\title{
Special issue on Ambient Intelligence
}

\author{
Satyen Mukherjee $\cdot$ Emile Aarts $\cdot$ Terry Doyle
}

Published online: 25 November 2008

(C) The Author(s) 2008. This article is published with open access at Springerlink.com

Ambient Intelligence (AmI) refers to predominantly electronic solutions that allow environments to be sensitive, adaptive, and responsive to the presence of people (Aarts et al. 2002). This opens up a world of unprecedented experiences to people in their day to day surroundings. Building on the early ideas of ubiquitous computing by Marc Weiser (1991) who envisioned a digital world where electronic devices are embedded to form a fine grained distributed network, AmI aims to go further in integration by involving the entire environment and any physical object in the interaction with people, thus improving their well being, productivity, creativity, and leisure through enhanced user system interaction.

Furthermore, beyond mere physical integration of electronics, this paradigm focuses on the creation of enhanced experiences, thereby having major cultural and business related implications (Aarts and Encarnaçao 2006; Aarts and Marzano 2003).

The notion ambience in Ambient Intelligence refers to the need for a large-scale embedding of technology in a way that it becomes unobtrusively integrated into every-day objects and environments. The notion intelligence reflects

S. Mukherjee $(\bowtie)$

Philips Research-North America,

345 Scarborough Road,

Briarcliff Manor, NY 10510-2099, USA

e-mail: satyen.mukherjee@philips.com

E. Aarts

Philips Research, High Tech Campus 34,

5656 AE Eindhoven, The Netherlands

T. Doyle

Philips Research, High Tech Campus 37,

5656 AE Eindhoven, The Netherlands that the digital surroundings exhibit specific forms of social interaction, i.e., the environments should be able to recognize the people that live in it, personalize to individual preferences, adapt themselves to the users, learn from their behavior, and possibly act upon their behalf.

This implies that embedding through miniaturization is the main systems design objective from a hardware point of view. From a software point of view we distinguish between the following major AmI functionalities, i.e., context awareness, ubiquitous access, and natural interaction. The user benefits of the AmI paradigm are aimed at improving the quality of peoples' lives by creating the desired atmosphere and functionality via intelligent, personalized, interconnected systems and services. However simple this requirement may sound, its true realization is for the time being not within our reach.

There are three global factors that enable the development of Ambient Intelligence, i.e., technology, global connectivity, and socio-economical aspects. Focusing on the technological factor, we use a well-known frame of reference provided by the developments in semiconductor industry, which is known as Moore's law (Noyce 1977). This law states that the integration density of systems on silicon doubles every 18 months. This trend is going on for more than 30 years and provides a clear forecast for the development of semiconductor technology. Recently, some new angles have been opened which in conjunction with Moore's law can be formulated as follows.

- 1D-Moore is the one-dimensional continuation of the classical Moore's law into the sub-micron domain of micro-electronics, resulting in small and powerful integrated circuits that can be produced at low cost.

- 2D-Moore is the development of two-dimensional large-area electronic circuitry of extremely low cost, 
possibly using other technologies than silicon such as polymer-electronics.

- 3D-Moore refers to the development of ultra-high functional three-dimensional circuitry consisting of Micro Electronic Mechanical Systems (MEMS) or Systems in a Package (SIPs) that integrate sensor, actuator, computing, and communication functions into a singe nano-electronics system.

Following the lines of thought imposed by the different developments of Moore's law one can conclude that the design and manufacturing of electronic devices has reached a level of miniaturization which allows the integration of electronic systems for processing, communication, storage display, and access into any possible physical object like clothes, furniture, cars, and homes, thus making people's environments smart. For extensive overviews of the various hardware developments that are relevant for Ambient Intelligence refer to (Mukherjee et al. 2006; Weber et al. 2005).

Basic devices for Ambient Intelligence AmI environments may consist of three types of devices, which are discriminated on the basis of their power consumption. This classification is justified by the general belief that power is the constraining factor in the distribution of AmI functionalities over an AmI environment (Basten et al. 2003; De Man 2005). This leads to the following device hierarchy.

- Autonomous devices that empower themselves autonomously over a full lifetime. They extract the required energy from the environment by scavenging light or electromagnetic energy, mechanical energy from vibrations or from temperature differences by means of thermoelectric generator. Examples are all kinds of tags and sensors.

- Mobile devices that use rechargeable batteries with typical autonomous operational times of a few hours, and standby time of several days. Examples are personal digital assistants, mobile phones, wireless monitors, portable storage containers, and intelligent remote controls.

- Stationary devices that have almost unlimited energy resource because they are empowered by a mains network. Examples are large flat displays, recording devices, (home) servers, and large storage and computing devices.

The energy availability as well as the power dissipation of a device may change over time. Different modes of operation may require different energy budgets, which may cause large variations in peak to average power dissipation. Evidently, for all types of devices introduced above energy management is the key factor in the determination of the amount of information processing that can be carried out by the individual devices. The different devices may come in different shapes and recent technological developments have given rise to many new devices called AmIware, that may become part of an integrated environment, ranging from autonomous sensors, and micro-camera's up to ebooks and 3-D displays; see Fig. 1.

These basic AmI functionalities apply to a large variety of environments and they can implement a variety of applications that are meaningful to people in their everyday-life activities such as work, security, healthcare, entertainment, and personal communications. They can be realized by optimally combining information processing technologies distributed over different device types, and the main issue is how to distribute the functionalities over the available devices. To shed some light on this we elaborate on each of these issues in slightly more detail.

Context awareness Context awareness is realized in three steps. Firstly, a wireless network of autonomous sensors is used to sense the environment. Many different sensors may be used to collect mechanical, physical, chemical, or biological data. Typical examples of such quantities are position, speed, acceleration, temperature, pressure, brightness, acid degree, magnetic flux, skin humidity, and heart beat. Secondly, the raw data are processed to represent relevant contextual information within a given contextual model. Combining the data and classifying them into higher-level data-items that represent states of the AmI environment may lead to the computation of new contextual information. This step is often referred to as sensor fusion and requires a computational effort that may be available in the autonomous devices if the processing is simple as in discriminating between day and night or sound and silence. It however becomes rapidly more demanding if more complex contextual states need to be classified like type or level of certain activities. In that case one must resort to implementations in mobile or stationary devices. Thirdly, interpretation and combination of states is required to extract novel information at a high semantic level using contextual models of the environment and specific behavioral patterns of people. This finally may lead to the deduction of desired pro-active responses of the AmI environment. These tasks involve on average major computational efforts, which may only be available within stationary devices.

Ubiquitous access Ubiquitous access requires the availability of digital media such as audio, video, pictures, and data at any time, any place. Access involves retrieving, streaming, and playing of media, which are computationally demanding tasks and therefore require mobile or stationary devices. Digital media require storage devices, which can either be local or embedded in a broadband communication network. Mobile devices can store data 


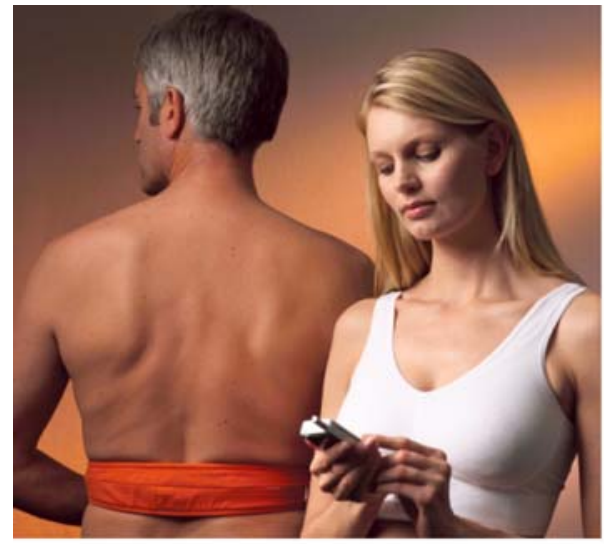

a.

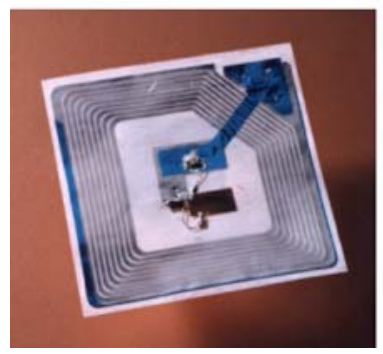

C.

Fig. 1 Examples of AmIware (AmI technologies). a Wearable Electronics. Electronics integrated into clothing can monitor vital body functions and can autonomously and wirelessly contact communication systems for readout or emergency rescue. b Photonic Textiles. Interactive light-emitting structures that are woven into fabric open up innovative lighting applications for atmosphere providing, illumination and indication. c Polymer Electronic RFID Tags.

locally using solid-state storage or disk technology. Networked storage is achieved through stationary server-type systems with large storage capacities. Networked terminal access is achieved by streaming data, preferably wirelessly, from a stationary storage device to a mobile or stationary device. Streaming high-quality video over wireless communication channels requires sophisticated compression and quality-of-service techniques. If the terminals are mobile devices, effective power management techniques are needed. Especially in the case where mobile devices need to be able to display several hours of high-quality video, major non-trivial performance requirements are imposed on the design of such devices.

Natural interaction Interaction technology may involve all device types. Tangible objects that implement interaction concepts for the control of AmI environments may be implemented as autonomous devices. For instance, nearfield communication technologies may be used to implement user interface concepts for the control of ambient lighting devices trough tagged colored objects. Inclusion of multi-modal interaction concepts, such as vision, speech and gesture recognition, involves the execution of computa-

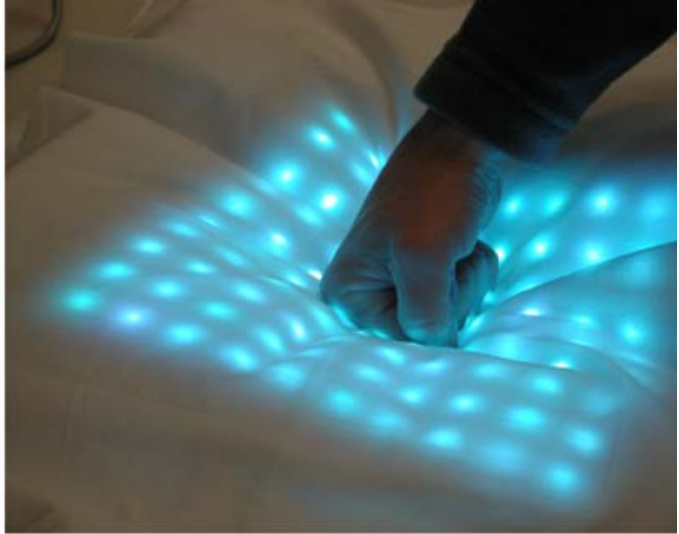

b.

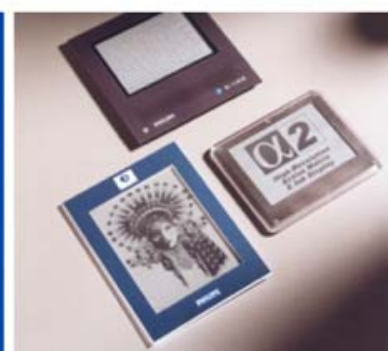

e.

Complete radio-frequency identification transponder integrated on an antitheft sticker. d Small Autonomous Networked Devices. A family of self-empowering electronic devices with standardized form factor applicable to a large variety of sensor functions. e Electronic Paper. High-resolution Electronic Ink Display with paper-like viewing characteristics, excellent contrast ratio, and clear images that can be seen from any angle

tionally demanding algorithms requiring mobile or stationary devices. If intelligence is added, computational efforts may range up to several Tops, which can only be achieved by using stationary devices. Examples of such interaction concepts are conversational interfaces, three-dimensional interactive video interfaces, and emotional robot interfaces.

Trends The device hierarchy described above introduces three major challenges for the design of AmI environments, which can be related to low-power design, design for distribution, and experience design, respectively. Below, we briefly elaborate on each of these challenges; see also (Basten et al. 2003; De Man 2005).

Low-power design Power limitations probably are going to impose the most demanding and challenging design objectives upon AmI environments, and this holds for all three types of devices.

Autonomous devices should use energy scavenging techniques applying micro-fuel cells, ultra capacitors, photovoltaic cells, vibrational converters, and radio-active power sources to empower themselves autonomously through physical environmental properties such as temper- 
ature gradients, light, wind and air flow, pressure variations, and movement (Rabaey et al. 2002).

Mobile devices require high-duty batteries with efficient and effective re-chargeability properties. Current battery technologies using zinc-air, lithium, or alkaline chemistry provide insufficient energy density capabilities if they are used to empower mobile devices that should be capable of playing several hours of high-quality video. Furthermore, the embedding of electronics in mobile devices such as wearables, tangible objects, and small furniture calls for batteries with non-conventional form factors that support foldable and bendable applications.

As AmI environments will contain several tens up to hundred stationary devices, classical empowering methods can no longer be used because of their thermal and environmental burdening. Consequently, new disruptive low-power technologies are needed. Asynchronous design paradigms and on-chip power management techniques are promising approaches but most probably new process technologies are needed which may be substantially different from the current CMOS technologies.

Design for distribution Design for distribution will call for novel middleware constructs that enable multiple networked and distributed applications and services to coexist and cooperate. The three main issues in this respect relate to interoperability, heterogeneity, and dynamics.

- Interoperability refers to the ability to exchange devices and application code in networked systems. The issue also includes the development of communication protocols that support plug and play such as HAVi, IEEE 1394, Bluetooth, UpnP, and IEEE 802.11.

- Heterogeneity refers to the ability to run software applications on devices with markedly different performance characteristics, i.e., mobile versus stationary or general purpose versus dedicated. This calls for middleware that is scalable to meet different footprints and operating systems. It also calls for bridging and gateway concepts that support data exchange between networks applying different communication protocols.

- Dynamics refers to the ability of networked systems to adapt themselves to changes of the environment, such as position, context, configuration, data, and others.

To be able to cope with the issues mentioned above, AmI middleware should support the following functionalities: device abstraction, device detection, resource management, streaming management, content and asset management, personalization, multi-modal interaction, context awareness, and security.

Evidently, design for distribution calls for the development of a middleware layer that is capable of gluing together the various devices in an AmI environment. For the individual devices the following major challenges can be formulated. For autonomous devices an operating system is required that can handle large wireless networks of distributed sensors and actuators. For mobile devices communication middleware is needed that supports device discovery and interoperability. For stationary devices streaming management and asset management are the key issues.

Experience design The design of AmI environments differs markedly from the design of classical single-box systems. AmI environments introduce new options for services and applications. The fact that no boxes will be present anymore introduces the need to come up with novel interaction concepts that allow the user to communicate with their electronic environment in a natural way.

As indicated above, AmI applications involve a broad range of technologies that are made possible with multidisciplinary research, as elaborated in a number of publications (Mukherjee et al. 2006; Weber et al. 2005).

In this special issue we address four aspects of the technology for Ambient Intelligence, which builds on a general concept for a distributed systems architecture consisting of different types of basic devices depending on their power consumption, their functionality, including security, personalized and collaborative service.

In a typical AmI application involving assisted living, for instance, one encounters a myriad of sensors and mobile devices flexibly embedded in the surrounding. Powering these devices seamlessly and effectively requires solutions that do not involve wiring to power outlets or even replacing exhausted batteries periodically. Instead, it is essential to provide a continuous source of power at the appropriate level with a long lifetime. Ophet Veld et al. (2009) addresses this issue and describes a solution for powering sensors based on mechanical energy scavenging for autonomous wireless sensor network applications including the required energy management and storage technologies.

Another aspect of an AmI application is the generation, wireless transmission and analysis of large quantities of sensitive information which needs to be protected against unauthorized eavesdropping by proper security measures including data encryption with secure keys. Guajardo et al. (2009) discusses the use of Physical Unclonable Functions (PUF) as secret keys in RFID systems.

In many AmI applications involving large scale networking, traditional centralized controls architecture can be compared with distributed computation and control through distributed collaboration for maximizing productivity. Also termed ubiquitous computing after Mark Weiser, this requires additional security and privacy protection considerations. Hwang and Yuan (2009) describes a collaborative iTrust e-service for exploring proximal collective wisdom in an ad-hoc ubiquitous environment. 
Extending AmI applications into the mobile environment raises additional computational challenges that need to be addressed. O'Grady et al. (2009) addresses the issues related to the deployment of intelligence in a mobile computing environment.

Open Access This article is distributed under the terms of the Creative Commons Attribution Noncommercial License which permits any noncommercial use, distribution, and reproduction in any medium, provided the original author(s) and source are credited.
Dr. Mukherjee has served as Department Head, Microelectronic Devices, Circuits and Systems at Philips Research, Briarcliff Manor, New York where he has conducted research in a number of semiconductor technologies, circuits and systems areas and applications in lighting, energy management, consumer devices, ultrasound imaging, wireless communications and displays. Prior to joining Philips Dr. Mukherjee worked on non volatile memory devices at Intel Corporation, and Exel Microelectronics, San Jose, California. Dr. Mukherjee holds a Ph.D. from Carleton University, Ottawa (1981) and a B.Tech degree from Indian Institute of Technology, Kharagpur, India (1976) where he was awarded the President of India Gold medal for top ranking in all branches of Engineering.

\section{References}

Aarts, E., \& Encarnaçao, J. (Eds.) (2006). True visions: tales on the realization of Ambient Intelligence. Berlin: Springer.

Aarts, E., \& Marzano, S. (Eds.) (2003). The new everyday: Visions of Ambient Intelligence. Rotterdam: 010.

Aarts, E., Harwig, H., \& Schuurmans, M. (2002). Ambient intelligence. In J. Denning (Ed.), The invisible future (pp. 235-250). New York: McGraw Hill.

Basten, T., Geilen, M., \& de Groot, H. (2003). Ambient Intelligence: impact on embedded system design. Dordrecht: Kluwer.

De Man, H., (2005). Ambient intelligence: gigascale dreams and nanoscale realities. International Solid State Circuits Conference, Digest of Technical Papers, pp. 29-35.

Guajardo, J., Skoric, B., Tuyls, P., Kumar, S. S., Bel, T., Blom, A. H. M. et al. (2009). Anti-counterfeiting, key distribution, and key storage in an ambient world via physical unclonable functions. Information Systems Frontiers, 11(1), (this issue).

Hwang, Y-C., \& Yuan, S-T. (2009). Ubiquitous collaborative iTrust service: exploring proximity collective wisdom. Information Systems Frontiers, 11(1), (this issue).

Mukherjee, S., Aarts, E. H. L., Ouwerkerk, M., Rovers, R., \& Widdershoven, F. (Eds.) (2006). AmIware: hardware drivers for ambient intelligence. Berlin: Springer.

Noyce, R. N. (1977). Microelectronics. Scientific American, 237(3), 63-69.

O'Grady, M. J., O'Hare, G. M. P., Chen, J., \& Phelan, D. (2009). Distributed network intelligence: a prerequisite for adaptive \& personalized service delivery. Information Systems Frontiers, 11 (1), (this issue).

Ophet Veld, B., Hohlfeld, D., \& Pop, D. (2009). Scavenging mechanical energy for ambient intelligent devices. Information Systems Frontiers, 11(1), (this issue).

Rabaey, Ammer, J., Karalan, T., Li, S., Otis, B., Sheets, M., et al. (2002). Pico-radio for wireless sensor networks: the next challenge in ultra low power design. International Solid State Circuit Conference, Digest of Technical Papers, pp. 200-210.

Weber, W., Rabaey, J., \& Aarts, E. (Eds.) (2005). Ambient intelligence. Berlin: Springer.

Weiser, M. (1991). The computer for the twenty first century. Scientific American, 165(3), 94-104.

Dr. Satyen Mukherjee is currently a chief scientist and senior director for research strategy in North America. Prior to this he was the ad interim managing director of Philips Research North America.
Prof. Dr. Emile Aarts is Vice President and Scientific Program Manager of the Philips Research Laboratories Eindhoven, The Netherlands. He holds an M.Sc. and Ph.D. degree in physics. For more than twenty-five years he has been active as a research scientist in Computing Science. Since 1991 he holds a position of part-time professor at the Eindhoven University of Technology, from 1991-2007 with the Faculty of Mathematics and Computing Science and since 2007 with the Faculty of Industrial Design. He also serves on numerous scientific and governmental advisory boards at national and European level. He is the co-author of more than ten books and more than two hundred scientific papers on a diversity of subjects including nuclear physics, VLSI design, intelligent algorithms and interaction design. He has been involved in the launch of the concept of Ambient Intelligence and in the foundation of Philips' ExperienceLab. His current research interests focus on interaction technology and design.

Dr. Terry Doyle received his $\mathrm{PhD}$ in Signal Processing from Trinity College, Dublin in 1983. Following 2 years as a lecturer, he joined Philips Research laboratories in 1984 where he carried out research into novel (especially flat) display systems in various functions with strong interaction with the business divisions. He moved to Taiwan in 1996 to set up an Innovation Centre that focused on advanced development in the areas of speech recognition, Internet appliances and storage systems. In 1998, he joined the Research Laboratory in Paris, where he was appointed Research Director of the Interactive Networked Systems sector in 1999 that focused on video coding and communication techniques-from IC's to applications. He was Managing Director of Philips Research UK from September 2002 until February 2008 and was responsible for the Worldwide Philips Research Connectivity Programme from 2003-2007. In 2007, he became programme manager for the Global Technology Programme with activities in the UK, USA, China, Germany and the Netherlands. In this role, there is a major focus on the Lighting research portfolio in addition to networked microsystems for sensing and actuation in the areas of Healthcare \& Lifestyle. Dr Doyle is a board member of the Technology Foundation in The Netherlands and is also a member of the Industrial Advisory board of IMEC, Belgium. 